\title{
CONTRIBUTO ALLA CONOSCENZA DEI LATINISMI E ROMANISMI ANTICHI IN SLOVENO ${ }^{1}$
}

\section{Romanizzazione, continuità e contatti linguistici slavo-romanzi sul territorio orientale delle Alpi e nell'area nordadriatica nel periodo paleoslavo}

Il fatto che il territorio dell'odierna Slovenia, soprattutto occidentale, rimanesse popolato dagli autoctoni di origine romanza ancora parecchio tempo dopo l'arrivo degli slavi è ormai indiscutibile. Quanti fossero, come suonasse il latino che parlavano, ${ }^{2}$ quando si assimilassero, in che ambiti della cultura materiale e spirituale si sentisse il loro influsso, sono invece questioni molto più complesse alle quali fino ai nostri giorni non si è potuto dare una risposta soddisfacente. Questo problema viene trattato da diverse scienze storiche e dalle loro discipline ausiliari: la storia con la storia del cristianesimo, l'archeologia con l'epigrafia e l'etnologia.

1. Osservazioni delle scienze storiche sulla romanizzazione, la continuità e i primi contatti slavo-romanzi

La storia insegna che l'occupazione romana dei nostri territori si svolse gradualmente, ${ }^{3}$ vale a dire che in certe regioni la romanizzazione era cominciata duecento anni prima che in altre. Il territorio dell'odierna Slovenia fu diviso in tre unità amministrative: il Norico, la Pannonia e la decima provincia dell'Italia (Venetia et Histria). Dobbiamo sottolineare che, a causa della sua posizione geografica, questo territorio rappresentava un collegamento fra la parte orientale e occidentale dell'impero romano, ed è dunque naturale che i Romani costruissero in quest'area una rete stradale abbastanza fitta. I collegamenti stradali e la rapida crescita economica con lo sviluppo

$1 \quad$ Nel presente saggio ci proponiamo di esporre sinteticamente i risultati della ricerca dal titolo Starejši latinski in romanski jezikovni fond $v$ slovenščini (Il fondo antico di origine latina e romanza in sloveno), tesi di master discussa presso la Facoltà di Filosofia di Lubiana il 23 gennaio 1997, relatore il prof. Mitja Skubic, correlatrice la prof.ssa Alenka Šivic-Dular.

2 Qui dobbiamo pensare pure al problema del sostrato.

3 Dall' occupazione dell' Istria poco dopo la fondazione di Aquileia (181 a.C.) fino al primo decennio d.C. 
dell'agricoltura, dell'allevamento del bestiame, della frutticoltura e della viticoltura da un lato e dall' altro il progresso dell'industria mineraria e dell' artigianato che comporta come conseguenza l'esportazione in Italia attraverso Aquileia ${ }^{4}$ il servizio militare obbligatorio nelle altre provincie dell'impero, l'urbanizzazione intensiva che si manifesta nella fondazione di unità amministrative e accampamenti militari, l'afflusso di militari e popolazione civile, soprattutto di mercanti e veterani, dall'Italia, il reclutamento della popolazione autoctona per il servizio militare, ${ }^{5} \mathrm{l}$ 'assimilazione o almeno il bilinguismo dei nobili della provincia, la migrazione nel terzo secolo della popolazione dalle città alla provincia, ${ }^{6}$ tutto questo avrebbe dovuto contribuire ad una romanizzazione relativamente rapida ed intensa. Non dobbiamo però dimenticare che certi fatti storici, comunque importanti per la densità della popolazione e per la sua struttura nazionale, sono stati interpretati in modi completamente diversi. ${ }^{7}$ In generale, gli studiosi sono tuttavia concordi nel considerare che sul territorrio delle Alpi orientali e delle Prealpi non si può parlare di una romanizzazione di carattere equilibrato. ${ }^{8}$

Sul carattere della romanizzazione in queste parti si potrebbe dedurre anche in base allo studio del materiale epigrafico. Le ricerche hanno dimostrato che la lingua delle iscrizioni latine trovate sul territorio dell'odierna Slovenia non comprova la presenza di innovazioni importanti rispetto alla norma e non differisce dal latino delle iscrizioni in generale. ${ }^{9}$ A questa constatazione non dobbiamo però attribuire un'importanza eccessiva visto che Haralambie Mihăescu è arrivato alla stessa conclusione dopo aver studiato a fondo il latino delle fonti scritte dell'Europa sudorientale. ${ }^{10}$ Secondo la sua opinione, condivisa da un gran numero di studiosi, nello studio della latinità di una certa regione l'importanza delle fonti epigrafiche è grande ma anche relativa perché non vi si riflettono in modo sufficiente la ricchezza e la varietà della lingua parlata. ${ }^{11}$ Perciò, ogni conclusione fatta sia in base al loro numero ${ }^{12}$ sia alla

4 Kos 1956: 18.

5 Mihăescu 1978: 42.

6 Jaro Šašel, "K poselitveni zgodovini in urbanizaciji Slovenije v antiki", ANS 68.

7 Cfr. le opinioni sulle conseguenze delle incursioni dei Quadi e Marcomani (169 d.C.) seguite da una grave epidemia della peste negli scritti di Grafenauer (Zgodovina 71) e Crevatin (1992: 213-214). Sono interessanti anche le differenze nelle interpretazioni della ben nota citazione di Velleio Paterculo ("In omnibus autem Pannoniis non disciplinae tantummodo, sed linguae quoque notitia Romanae, plerisque etiam litterarum usus et familiaris animorum erat exercitatio"; II 110, 5) proposte da Grafenauer (1954: 68), Mihăescu (1978: 42), Dahmen (1992: 111) e Crevatin (1992: 214).

8 Il Bezlaj parla di romanizzazione superficiale ("površna romanizacija"; Bezlaj 1967: 94), il Crevatin usa il termino romanizzazione settoriale (Crevatin 1992: 212).

9 Cfr: Skubic 1981.

10 "Pour nombreux et variés qu'ils soient, les faits linguistiques conservés dans les inscriptions et les textes du sud-est de l'Europe ne sont pas spécifiques pour cette région, ni uniques. Pour chacun d'entre eux, nous nous sommes efforcé de trouver des phénomènes analogues en Occident et nous y sommes presque toujours parvenu..." (Mihăescu 1978: 327).

11 Cfr. Mihăescu 1978: 327, similmente Gaeng 1987: 79. Le ragioni all'origine di questa situazione nel latino scritto dell'Europa sudorientale sarebbero, secondo Mihăescu, la tradizione epigrafica, 
loro innovatività (o all' assenza della stessa) ${ }^{13}$ può dimostrarsi molto ingannevole, ci possono tuttavia offrire informazioni utili almeno i dati sul tempo e sul luogo della loro origine.

Secondo l'opinione degli storici sloveni la continuità dell'antichità sul territorio dell'odierna Slovenia fu di carattere piuttosto superficiale, ${ }^{14}$ ma siccome colonizzarono soprattutto le regioni già popolate seguendo spesso le correnti dei fiumi, ${ }^{15}$ gli slavi alpini incontrarono al loro arrivo senza dubbio gli abitanti di origine romanza o romanizzati chiamati "Valacchi (Vlahi)". ${ }^{16}$ Lo studio fonetico di certi toponimi di origine preslava in sloveno dimostrerebbe che questi "pastori a metà romanizzati"17 vissero tra gli slavi alpini almeno fino al decimo secolo. ${ }^{18} \mathrm{Gli}$ esperti ritengono che si trattò soprattutto di agricoltori e di pastori. Per Milko Kos non si può parlare di un influsso diretto della cultura spirituale antica o di una continuità politicoamministrativa, anche se, nello stesso tempo, non si dovrebbe sottovalutare l'influsso degli autoctoni sulla struttura fisica e spirituale degli immigrati sloveni. Gli aborigeni avrebbero inoltre trasmesso agli slavi alpini numerose acquisizioni della cultura materiale, soprattutto nell'ambito dell'agricoltura, dell' alpeggio, dello sfruttamento minerario e dell' artigianato. Trattando di questi problemi, il Kos si rifà, tra l'altro, ai reperti degli scavi archeologici e sottolinea il fatto che gli oggetti trovati nelle tombe paleoslave dell'ottavo e nono secolo mostrano nelle loro forme l'influsso della tradizione antica. ${ }^{19}$

I fatti storici che attirano la nostra attenzione sono in primo luogo quelli legati alla storia del cristianesimo che non solo ha giocato un ruolo importante nella romanizzazione ma ha avuto importanza pure per la continuità dell'antichità in

l'immigrazione della popolazione latinofona fino all'inizio del quinto secolo e l'espansione del cristianesimo (ibid.).

12 Su questo punto dobbiamo attenerci all'osservazione di Herman secondo la quale il numero delle iscrizioni trovate non può riflettere la situazione reale poiché gran parte del materiale epigrafico è stata distrutta (Herman 1976: 9).

13 Herman ha constatato che le deviazioni dalla norma nelle iscrizioni del Norico sono rarissime il che sarebbe proprio la prova della superficialità della romanizzazione di questo territorio poiché "...la bonne tenue linguistique des inscriptions est simplement le signe du caractère isolé de la présence romaine, d'une absence presque complète de romanisation parmi les indigènes, entraînant la survivance de schémas orthographiques et linguistiques rigides." (Herman 1976: 14).

14 Cfr. Kos 1985: 120.

15 Cfr. Kos 1985: 118.

16 Cfr. Kos 1985: 121-132 ("Vlahi in vlaška imena med Slovenci", per la prima volta in: Glasnik Muzejskega društva za Slovenijo, 20, 1939, 226-235). Per l'etimologia v. ESSJ Il: $119-120$ (s.v. Lah).

17 Bezlaj 1967: 32.

18 Ibid. 94.

19 Kos 1985: 119. Per i risultati degli scavi che potrebbero testimoniare dell'influsso dell'antichità sulla cultura materiale degli slavi su questo territorio cfr. la dissertazione di Paola Korołec con il titolo Zgodnjesrednjeveška arheološka slika karantanskih Slovanov (L'immagine archeologica degli slavi di Carantania nel primo medioevo; Korošec 1979, passim). 
generale. Il problema della continuità del cristianesimo sul territorio delle Alpi orientali e nell' area nordadriatica è stato trattato da un gran numero di studiosi che fino ai nostri giorni non ha potuto però trovare una soluzione generalmente accettabile. Stando a Rajko Bratož il problema è senza dubbio uno dei più ardui nello studio del cristianesimo nel periodo di transizione dall'antichità al medioevo e si potrà risolvere soltanto con una ricerca interdisciplinare. ${ }^{20}$ Visto lo stato delle ricerche sulla tarda antichità e sul primo medioevo da noi, ${ }^{21}$ si potrebbe presumere lo stesso per il problema della continuità in generale. ${ }^{22}$

\section{Prove linguistiche della continuità e del interazione slavo-romanza nel periodo paleoslavo}

Il periodo detto paleoslavo di cui stiamo parlando, vale a dire quello che inizia coll'arrivo degli slavi e dura approssimativamente fino alla fine dell'undicesimo secolo, ${ }^{23}$ fu di importanza cruciale tanto per la sfera linguistica slava che per quella romanza: si tratta per tutt'e due - prima per quella romanza e un po' più tardi per quella slava - di un periodo di transizione in cui dalla base linguistica comune, ossia dal latino volgare nel primo e dal paleoslavo nel secondo caso, si sono sviluppate poco a poco le lingue romanze o slave. Sullo sviluppo dello slavo alpino influiscono da una parte il processo di mescolanza e confluenza delle differenti correnti d'immigrazione e dall'altra gli importanti cambiamenti sociali tra i quali dobbiamo menzionare il cambiamento dell'assetto sociale, la cristianizzazione, la perdita dell'indipendenza politica e l'insediamento fisso. ${ }^{24}$ Dall'undicesimo secolo in poi possiamo già parlare della fase di fondamentale dialettalizzazione dello sloveno caratterizzata dai mutamenti che in un relativamente breve periodo di pressapoco due secoli danno origine a notevoli differenze dialettali. ${ }^{25} \mathrm{Dal}$ decimo secolo in poi il territorio dell'odierna Slovenia è esposto ad un'intensa colonizzazione germanica. Prevale quindi l'influsso linguistico germanico a cui nel periodo seguente lo sloveno è esposto molto di più che a quello romanzo (eccezion fatta dell'estremo Ovest). Non si deve tuttavia trascurare il fatto che gli slavi alpini furono in contatto non solo con gli autoctoni romanizzati ma pure con gli abitanti delle regioni vicine dove in questo periodo si stavano formando vari dialetti romanzi: il friulano, l'istroromanzo e l'ormai estinto dalmatoromanzo. Sui contatti tra

21 Riesaminando la situazione su questo campo, l'archeologo Jaro Šašel (ANS 68.69) lamenta tanto la scarsità delle fonti scritte e dei reperti archeologici come la mancanza di sistematicità delle ricerche nelle altre discipline scientifiche.

22 Per il problema della continuità v. soprattutto Alpes Orientales V (AO); Bezlaj 1961, 1967, 1969; Grafenauer 1969 e 1988, Kos 1985, Crevatin 1992, Dahmen 1992.

23 Cfr. II.1.

24 Si tratta dunque di un periodo limitato tanto dal punto di vista linguistico che di quello storico (cfr. Grafenauer 1969: 63 e 1988: 345).

25 Logar 1965: 5. Cfr. pure Bezlaj 1967: 111. 
la popolazione romanizzata e gli slavi alpini ci forniscono qualche informazione i toponimi sloveni di origine romanza e i prestiti antichi in sloveno.

\subsection{Importanza dei toponimi}

I nomi di luogo possono causare parecchi problemi per l'arcaicità delle parole base che rende oscuro il loro significato ${ }^{26}$ e la diversità delle forme in cui ci sono pervenuti. È difficile perciò ricostruire la forma base e le leggi fonetiche che agirono al momento del prestito. ${ }^{27}$

Per stabilire il limite tra le parti sudorientale e nordoccidentale della Romània, gli studiosi hanno tenuto conto tra l'altro delle caratteristiche fonetiche dei toponimi di origine latina in quest'area: ${ }^{28}$ il loro vantaggio sugli altri prestiti consiste nel fatto che il luogo del prestito è conosciuto. I toponimi di questo tipo, di grande importanza per il linguista, si incontrano però raramente sul nostro territorio. ${ }^{29}$ Prevalgono le basi toponomastiche prelatine che si conformano tuttavia alle leggi fonetiche del latino volgare,${ }^{30}$ mentre le tracce dei toponimi con le tipiche finali latine sono poche.

\subsection{Importanza dei prestiti antichi}

Il primo ad aver richiamato l'attenzione sull'importanza dei latinismi e dei romanismi antichi nelle lingue slave meridionali per lo studio del cosiddetto latino balcanico è stato Petar Skok. ${ }^{31}$ Secondo il Bezlaj soltanto l'accurato studio dei periodi più antichi della simbiosi linguistica slavo-romanza permetterà agli studiosi dello sloveno di stabilire le differenze culturali tra gli autoctoni e gli immigrati slavi, di comprendere il processo di fusione delle due civiltà e di tracciare finalmente il limite tra il substrato ed adstrato, di fare dunque un' analisi globale degli influssi culturali stranieri sugli slavi alpini. ${ }^{32}$ Constatando tanto l'uniformità linguistica che il carattere "urbano, libresco ed astratto" delle fonti scritte dell'Europa sudorientale, ${ }^{33}$ Haralambie Mihăescu propone un metodo di ricerca che consisterebbe nell' analisi approfondita

26 Spesso la parola che ha servito di base per la denominazione non si usa più nella lingua.

27 Cfr. Bezlaj 1967: 86. Per i nomi di luogo che confermano l'esistenza delle colonie degli aborigeni o almeno dei loro resti nel periodo dell'insediamento degli slavi cfr. Kos 1956:10-17; 1985: 130 e soprattutto F. Truhlar, "Krajevna imena gradišče, gomila, groblje, žale", ANS 106-112 (con la lista alfabetica delle varianti toponomastiche). Per i nomi dei fiumi v. SVI I: 5-17. Cfr. inoltre Bezlaj $1961,1969$.

28 Cfr. Grad 1965.

29 Bezlaj 1967: 94.

30 Ibid.

31 Skok 1940: 129.

32 Cfr. Bezlaj 1967, specialmente 84 e 102.

33 "Elles sont ... l'expression d'une certaine couche sociale peu familiarisée avec les réalités complexes liées étroitement aux occupations quotidiennes fondamentales: l'agriculture et l'élevage." (Mihăescu 1978: 328). 
degli elementi latini in tutte le lingue di questa zona tra le quali menziona esplicitamente pure lo sloveno. ${ }^{34}$ Per fare un' analisi di questo tipo bisogna tuttavia saper riconoscere questi elementi distinguendoli dagli altri prestiti, sia di quelli assimilati magari in un periodo antico ma con mediazione germanica, sia dai romanismi o croatismi più recenti.

\section{Ricerche dell'antico fondo latino e romanzo in sloveno}

Quanti di noi studiano gli influssi lessicali romanzi nello sloveno si rendono conto che parecchio rimane ancora da fare in questo campo, soprattutto se si pensa all'accurato ed esauriente lavoro di Hildegard Striedter-Temps sui germanismi in sloveno. ${ }^{35}$ Finora non c'è stata una rassegna di romanismi che includesse le indicazioni sul periodo del prestito come neppure una descrizione di sostituti sloveni per ogni suono romanzo nei vari periodi. Le voci slovene di origine romanza, tanto quelle assimilate nel periodo che ci interessa che quelle più recenti, vengono trattate sia nel quadro dei lavori generali, ${ }^{36}$ nei dizionari etimologici e nei dizionari dei prestiti nello sloveno e nelle altre lingue slave, ${ }^{37}$ sia dal punto di vista di un cambio fonetico ${ }^{38} \mathrm{o}$ nei limiti di un determinato campo semantico. ${ }^{39}$ Lo Skok ha indubbiamente grandi meriti nello studio dei contatti slavo-romanzi, ma l'oggetto principale delle sue ricerche, ad eccezione di qualche contributo minore nella maggior parte dei casi di carattere toponomastico, ${ }^{40}$ non è stato lo sloveno benché preso conseguentemente in considerazione nel suo materiale per il dizionario etimologico ed altrove.

L'unico importante lavoro dedicato interamente ed esclusivamente ai romanismi in sloveno è Romanische Lehnwörter im Slowenischen, una tesi dattiloscritta di 122 pagine e difesa dall'autore Manfred Mende nel 1950 alla Facoltà di filosofia dell'università di Berlino, relatore Max Vasmer. ${ }^{41} \mathrm{Si}$ tratta di una rassegna dei prestiti romanzi in sloveno dai più antichi ai più recenti. Il fondo lessicale antico di origine latina o romanza vi è rappresentato solo in parte e in un modo discutibile. Lo stesso si potrebbe dire per la classificazione dei romanismi impiegatavi. ${ }^{42}$

34 "Il s'impose donc nécessairement, comme méthode, d'élargir le champ d'observation, de recueillir le plus de matériel comparatif possible du reste du monde romain, de toutes les sources possibles; d'analyser comparativement, avec la plus grande attention, les éléments latins des langues roumaine, dalmate, albanaise, slovène, serbo-croate et bulgare." (ibid.). Il risultato delle sue ricerche su questo campo è il saggio La romanité dans le Sud-Est de l'Europe, Bucarest, 1993 (v. la recensione di Žarko Muljačić in: $Z R P h, 113 / 3,1997,471-474)$.

35 Deutsche Lehnwörter im Slovenischen (DLS).

36 Da menzionare i Latinismi e romanismi antichi nelle lingue slave meridionali di Luciano Rocchi (LRA).

37 Per esempio Štrekelj 1890 e 1892.

38 Per esempio Šturm 1927 (in italiano: Šturm, 1932-33) e 1928; Grad 1969.

39 Ultimamente Ferluga-Petronio 1980 e 1981; Cossuta 1995.

40 Cfr. Skok 1921, 1928, 1931.

41 V. RLS nella bibliografia. 


\section{Latinismi e romanismi antichi nel Dizionario della lingua letteraria slovena}

\section{Oggetto della ricerca e giustificazione del metodo}

Il nostro contributo allo studio dei contatti linguistici slavo-romanzi è un saggio ${ }^{43}$ nel quale abbiamo esaminato almeno uno strato di romanismi, quello più antico, limitandoci però alle parole che si sono conservate in sloveno fino ai nostri giorni e che si sono talmente acclimatate nella lingua da venir incluse nello Slovar slovenskega knjižnega jezika (Dizionario della lingua letteraria slovena). ${ }^{44}$ Formano l'oggetto della nostra ricerca circa una settantina di latinismi e romanismi basati su appellativi latini ${ }^{45}$ e assimilati con molta probabilità nel periodo denominato dagli slavisti "preistorico" 46 o "paleoslavo". ${ }^{47} \mathrm{Ci}$ siamo ingegnati a datarli quanto più esattamente possibile cercando di delineare i limiti spesso praticamente indistinguibili tra i prestiti più antichi e quelli che appartengono ad un periodo più recente. A questo scopo abbiamo rivolto una particolare attenzione alla loro immagine fonica e alla loro diffusione sul territorio slavo e nelle aree limitrofe. Dobbiamo però renderci conto che si può parlare di un latinismo o romanismo antico soltanto nel caso che la parola sia stata presa in prestito direttamente $^{48}$ dal latino o da una parlata romanza prima della fine dell'undicesimo secolo. I prestiti indiretti seppure antichi (come per esempio meníh $<$ aat. munih $<$ MŎNĂCHUS, REW $5654^{49}$ ) non sono oggetto della nostra ricerca perché non ci dicono niente sugli eventuali contatti tra gli abitanti romanzi (o romanizzati) e gli immigrati slavi. Per la stessa ragione abbiamo cercato di escludere le parole che originariamente sono romanismi antichi, ma che in realtà, dal punto di vista dello sloveno, rappresentano dei croatismi più o meno recenti. ${ }^{50}$ Abbiamo fatto eccezione soltanto per gli eventuali croatismi sulla cui origine non è stata finora accettata una soluzione etimologica definitiva.

42 Per una critica più dettagliata v. Bezlaj 1968-69.

43 Šega 1996.

44 V. SSKJ nella bibliografia.

45 Non rientrano dunque in questa ricerca gli appellativi sloveni basati sugli etnonimi, $\mathrm{i}$ toponimi e gli antroponimi di origine latina o romanza eccezion fatta per i casi dove questi si citano come esempi per qualche fenomeno fonetico.

$46 \quad$ Nahtigal 1952: 7.

47 Lamprecht 1987: 14.

48 "Contrariamente ai principi che reggevano gli studi dei neogrammatici noi oggi diamo precedenza all'etimologia prossima." (Muljačić 1978: 413). Questo è pure il metodo adottato da Hildegard Striedter-Temps nel suo saggio sui germanismi in sloveno (DLS).

49 Cfr. DLS 180.

50 Fanno parte di questo gruppo soprattutto gli ittionimi e le altre espressioni relative al mare (p.e. BOCA, REW $1182>$ cr. bükva > sln. búkva; cfr. ERHSJ I: 232). 
Tanto la mancanza delle fonti scritte quanto i numerosi problemi legati all'arrivo degli slavi e la continuità sul territorio delle Alpi orientali e dell'Adriatico settentrionale non ci permettono di farci un'idea precisa sulla situazione linguistica in quest'area nei primi secoli dopo l'immigrazione. Contribuisce inoltre alla confusione la mancanza di manuali indispensabili come per esempio una grammatica storica e un dizionario etimologico del friulano.

Al contrario dei prestiti recenti, questo tipo di prestiti sono difficilmente riconoscibili. Per questa ragione, non poche etimologie sono state stabilite soltanto recentemente, ${ }^{51}$ altre poi non si possono ancora spiegare in un modo soddisfacente. Le basi latine non sono più discutibili già da molto tempo, ${ }^{52}$ ma in certi casi è molto difficile o praticamente impossibile stabilire se si tratti di un prestito diretto dal latino o se si debba parlare di un prestito indiretto per mediazione sia del tedesco ${ }^{53}$ sia perfino di una delle lingue di sostrato poco conosciute. ${ }^{54}$ I problemi non si propongono dunque quando si vuole stabilire l'etimo latino di un certo romanismo antico, ma soltanto quando si cerca di determinare l'età del prestito e la forma originale nella quale è stato assimilato.

Se il materiale linguistico di cui disponiamo non ci fornisce dati sufficienti per stabilire esattamente la base etimologica e il periodo del prestito, ${ }^{55}$ ci può fornire delle informazioni utili 1'area in cui è diffuso. Il fatto che l'etimo si sia conservato nelle lingue romanze limitrofe, nelle altre lingue slave meridionali e in albanese come prestito diretto oppure in ungherese come prestito antico dallo slavo, potrebbe rappresentare un argomento a favore dell'antichità del prestito. Facendo conclusioni sulla base dell' area, dobbiamo però prendere tutte le possibili cautele: gli stessi etimi latini assimilati in periodi differenti, magari con la mediazione di altre lingue, hanno dato risultati simili di modo che è molto difficile distinguere tra loro i vari strati di prestiti.

51 Per esempio quella dello sln. žóga che risale a JǑCUS (REW 4587) con la mediazione del veneziano. Cfr. Bezlaj 1967: 59.

52 Si può, tuttavia, trovare qualche parola slovena di evidente origine straniera la cui forma originale non è ancora conosciuta. Bezlaj spiega per esempio la parola tolmún (it. "tonfano") come un romanismo antico derivandola dapprima dal latino volgare *TURBINIO "Wirbelwind" (REW 8995; SVI I: 100), poi dalla forma supposta *TULMINIONEM (Bezlaj 1967: 24; cfr. anche ibid., 32). La parola si rivela estremamente problematica, soprattutto perché esistono pure le varianti cemûn (Plet. I: 78), komun (SVI, ibid.), tomun, tomína e tomina (Plet. II: 67) e perché secondo l'opinione di certi etimologi non è da escludere completamente il legame con il toponimo Tolmin. Per la bibliografia relativa a questi problemi v. SVI I: $99-100$ e II: 263-264. La parola non figura nel nostro elenco per l'incertezza dell'etimologia.

53 Come per esempio sln. čebúla (CĒPǓLLA, REW 1820) o sln. rúta (ROTA, REW 7470). Cfr. Šega 1996 ss.vv.

54 Cfr. per esempio bek (ESSJ I: 16).

55 Il Popović (1960; 591-592) cita per il serbocroato esempi nei quali l'immagine fonica non può offrire nessun argomento a sostegno dell'ipotesi - secondo lui molto probabile - sull'antichità del prestito. 
Dati questi precedenti non dobbiamo stupirci se per più di un presunto antico romanismo si è trovato soltanto più tardi che si trattava di un romanismo più recente 0 perfino di un germanismo. ${ }^{56}$ Per certe parole, considerate ancora poco fa come latinismi o romanismi antichi, si sono fatte pertanto valere le spiegazioni sulla base di etimi paleoslavi. ${ }^{57}$

Da tutto questo risulta con evidenza che le conclusioni qui esposte devono essere considerate piuttosto come provvisorie, sopprattutto perché abbiamo lavorato su un fondo di parole limitato. Eventuali e più approfondite ricerche sulla base del lessico sloveno nel suo insieme potranno portare a correzioni e aggiunte.

\section{I sostituti dei suoni latini nello slavo alpino}

Nello studiare dal punto di vista fonetico il fondo di parole che abbiamo raccolto, abbiamo concentrato la nostra attenzione su due problemi. In primo luogo abbiamo cercato di stabilire i sostituti dei suoni romanzi nello slavo alpino ed i loro esiti nello sviluppo ulteriore dello sloveno. In secondo posto ci ha interessato in che misura le regole di sostituzione che abbiamo stabilito si accordano con quelle fissate precedentemente in base allo studio sia del materiale lessicale ${ }^{58}$ e dei toponimi ${ }^{59} \mathrm{di}$ origine latina o romanza sia dei prestiti antichi dalle altre lingue in sloveno. ${ }^{60}$ Dobbiamo però richiamare l'attenzione su alcune restrizioni per cui in base al materiale lessicale che abbiamo alla nostra disposizione non si devono fare delle conclusioni precipitose.

Anzitutto va considerato il fatto che nel processo di assimilazione di un prestito la lingua ricevente non sostituisce sempre i suoni della lingua d'origine con i suoni acusticamente corrispondenti semplicemente perché non fanno parte del suo sistema fonologico. La lingua che compie il prestito sostituisce dunque il suono sconosciuto con il suono che se le avvicina di più nel suo sistema fonologico. Sarebbe dunque sbagliato concludere sulla natura di un suono studiando esclusivamente i suoi sostituti nelle altre lingue.

Inoltre, parlando del sostrato preslavo nel lessico sloveno, France Bezlaj attira la nostra attenzione su un numero relativamente elevato di sviluppi fonetici irregolari. ${ }^{61}$ Nella sua critica del saggio di Vojmir Vinja, Jadranska fauna. Etimologija $i$ struktura naziva, Pavao Tekavčić approva l'idea della priorità del contenuto sul piano dell'espressione sottolineando che "...la regolarità dell'evoluzione fonetica, le leggi

56 Cfr. Šturm 1927: 52 e ESSJ III: 8 s.v. pápež.

57 Cfr. sIn. pastír. L'origine latina (cfr. PASTORE, REW 6279) è respinta da Metka Furlan (ESSJ III: 13).

58 Cfr. RLS.

59 Cfr. Bidwell 1961.

60 Si pensa qui soprattutto ai germanismi antichi. Cfr. DLS.

61 Bezlaj 1967: 96. 
fonetiche ecc. sono in molti casi secondarie e non riescono a spiegare evoluzioni complesse.". ${ }^{62}$ Tuttavia lo stesso autore avverte a ragione: "Siamo del parere che nessun cambiamento, per 'strano', isolato, sporadico che sia, avvenga nel modo del tutto arbitrario." 63 Il problema sta naturalmente nel fatto che è molto difficile stabilire i fattori che hanno causato le deviazioni per un periodo così remoto come il nostro.

Vorremmo menzionare ancora il fatto che tra le parole raccolte non è per niente trascurabile il numero di quelle che si possono annoverare fra i latinismi e romanismi antichi soltanto con una certa riserva: vuol dire che per ognuna di loro esiste più di una proposta etimologica, che le soluzioni etimologiche attuali sollevano dei dubbi e che sulla base dei dati con cui disponiamo in questo momento non è sempre possibile fare delle conclusioni sicure sull'origine e sull' antichità del prestito. Inoltre, la scarsità degli esempi per la sostituzione di alcuni fonemi ${ }^{64}$ accresce la complessità del problema.

\subsection{Vocali}

\section{a) $\bar{\imath}$ del lc. $>i$ del lv.:}

In posizione accentata questo suono viene sostituito nello slavo alpino con $i$ che dà in sin. $i$ nei prestiti víno (VİNUM, REW 9356), mlín (MŎLİNU, REW 5644) o eventualmente con $b$ in kómen (CAMİNUS, REW 1549) e lúben (LŬPĨNUS, REW 5170). ${ }^{65}$ Per la $i$ non accentata non abbiamo trovato nessun esempio tra gli etimi esaminati. 66

\section{b) $\breve{l}$ del lc. $>e$ del lv.:}

Il risultato nello salp. è $b$ che in sloveno si vocalizza o cade a seconda dell'intonazione e dalla sua posizione nella parola: măša (MĬSSA, REW 5610), pôper ${ }^{67}$ (PĬPĔRE, REW 6521), brósk/val (BRASSĬCA, REW 1278). ${ }^{68}$ Abbiamo dunque da fare con lo stesso sostituto che nel caso della $i$ breve del aat. ${ }^{69}$ Nello sln. krizma (CHRĬSMA, REW 1887) la $i$ è sostituita con la $i$ slovena (< salp. $i$ ). Esempi paralleli si possono trovare pure nei germanismi antichi, per esempio meníh dal aat. munih. $^{70}$

Tekavčić 1987: 168.

Ibid. 170 .

64 Cfr. 2.1 a), c), d) e g).

65 Nel primo caso si tratta molto probabilmente di un croatismo mentre nel secondo non è da escludere la mediazione del medio alto tedesco (cfr. DLS 155).

66 Per questo fonema si cita generalmente l'esempio CIVITATE > čuv $\iota d a d z>C ̌ e d a d$ ossia cuvbtatz> Cavtat. Cfr. Ramovš 1936: 34; Bidwell 1961: 116.

67 Per l'esito o v. Ramovš 1936: 165.

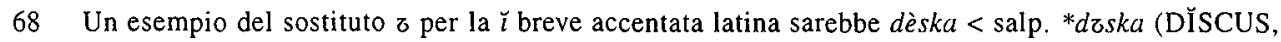
REW 2664; cfr. Ramovš 1936: 160), però la presenza di $z$ in questo prestito non sembra del tutto sicura (cfr. ESSJ I: 98).

69 Cfr. aat. scugin(a) > sln. skedènj (DLS 6).

70 Ibid. Nei prestiti dell'ambito della terminologia cristiana non dobbiamo escludere l'influsso delle 


\section{c) $\breve{e}$ del lc. $>$ in posizione tonica: lv. $e$ in posizione atona: lv. $e$}

Nella sillaba accentata questo suono viene sostituito con salp. $e$ che dà in sloveno $e$ : cèr (Č̆RRUS, REW 1838). Il sostituto della $e$ non accentata appare soltanto in pôper < salp. *pspьrb (PĬPĔRE, REW 6521). La semivocale slovena si sarebbe sviluppata in questo caso dallo salp. $b,{ }^{71}$ ma non si può dire niente di certo perché in sloveno questo risultato potrebbe dipendere ugualmente da una forma sincopata romanza. ${ }^{72}$

\section{d) $\bar{e}$ del lc. $>\underline{e}$ del lv:}

Nella posizione accentata abbiamo incontrato il sostituto salp. $i>\sin$. $i$ in golída (GALLETA, REW 3656), míza (MENSA, REW 5497), robída (RŬBĒTUM, REW 7407) ${ }^{73}$ L'esempio čebǔla (CEPǓLLA, REW 1820) parlerebbe in favore del sostituto $e$ per questo suono in posizione atona. Si tratta però di un prestito, assimilato molto probabilmente con la mediazione germanica. ${ }^{74}$

\section{e) $\bar{a}, \breve{a}$ del lc. $>a$ del lv.:}

Nei prestiti più antichi lo slavo alpino conosce due sostituti differenti per questa vocale in dipendenza dall' accento e dalla sua posizione nella parola. La $a$ accentata del latino volgare nella penultima sillaba viene sostituita nello slavo alpino con $a(\sin . a)$ in oltár (ALTĀRE, REW 381), bánja (*BANĔUM, REW 916), pogán (PĂGĂNUS, REW 6141), sák (SACCUS REW 7489), ecc. mentre nell' antepenultima la stessa vocale risulta nello slavo alpino in $\breve{a}$ che passa dopo a $o$ : brósktval (BRASSĬCA, REW 1278). Si pensa in genere che la differenza sia dovuta ad una pronuncia più breve della vocale in questa posizione. ${ }^{75}$ I prestiti con il sostituto $a$ per la $a$ accentata nell'antepenultima sillaba possono dunque essere considerati come più recenti, per esempio járbol (ARBŎRE, REW 606). Il sostituto $o(<\breve{a}$ ) appare nei prestiti più antichi pure per la $a$ non accentata: golída (GALLETA, REW 3656), ločíka (LACTUCA, REW 4833). Per quanto riguarda il prestito račún (RATIŌNE, REW 7086) è quindi da presumere che non risalga al periodo più antico. ${ }^{76}$

\section{forme dotte.}

71 Questa è la spiegazione di Bidwell (1961: 117, n. 21).

72 Secondo Bidwell, accanto a b (p.e. GLEMONA > glbmynz > Humin; cfr. pure Ramovš, 1936: 36), un altro sostituto per la $\breve{e}$ non accentata sarebbe salp. $e$, di cui finora si conosce un solo esempio, il toponimo NEVESTE > Nevest (Bidwell 1961: 117).

73 Per Ramovš, il sostituto salp. $\check{e}$ come nell'esempio Oglej < oglějb < AQUILEIA sarebbe la conseguenza di una pronuncia arcaica di questo fonema nelle parole dotte (Ramovš 1936: 40). Per il sopra menzionato sostituto $i$, cfr. ibid.; Bidwell 1961: 116.

74 Cfr. LRA 1820; Šega 1996: 52-54. Per la sostituzione della ẹ atona del lv. (< lc. e) v. pure sopra, n. 72 .

75 Cfr. Bidwell 1961: 115. Le forme con la vocale in iato del tipo BA(L)NĚUM (REW 916, sln. bánja), CALCEA (REW 1495, sln. hláče) avevano naturalmente già perduto una sillaba.

76 Cfr. però le varianti con $o$ nei dialetti croati occidentali (ERHSJ III: 93). 


\section{f) $\check{o}$ del lc. $>$ in posizione tonica: $o$ del lv. \\ in posizione atona: $\boldsymbol{o}$ del $\mathrm{lv}$.}

Gli esempi come vŕt < salp. *vъrtъ (HŎRTUS, REW 4296) o tŕta < salp. *tъrta (TŎRTA, REW 8809) dimostrano che questo fonema è stato sostituito nello slavo alpino con 6 . Dobbiamo però ammettere che in tutti e due i casi la vocale si trova in sillaba chiusa dove sembra che il suo timbro fosse più chiuso e più breve. ${ }^{77} \mathrm{Del}$ sostituto 6 si potrebbe parlare anche nell'esempio di kmèt (CŎMĬTE, REW 2078). ${ }^{78} \mathrm{~A}$ volte si possono trovare altri sostituti, come per esempio in sóld (SŎLĬDUS, REW 8069), che può essere spiegato come un prestito meno antico, o in ólje (ǑLEUM, REW 6054), assunto con verosimile mediazione germanica. ${ }^{79} \mathrm{Il}$ nostro materiale presenta un altro sostituto, cioè sln. $u$ in skúta (*EXCŎCTA, REW 2977), ljúlj/ka/ (LŎLIUM, REW 5112). Nella posizione non accentata la vocale si sostituisce con salp. $o$, che dà $o$ in sloveno: pogáča (FŎCACEA, REW 3396), óblat (ŎBLĀTA, REW 6012). Nell'esempio mlín < salp. *molinz (MŎLINUS, REW 5644) appare pure il sostituto 3.80

g) $\bar{o}$ del lc. $>\rho$ del lv.:

Si ritiene in generale che nella posizione accentata questo suono si sostituisca con $u$ lunga slava ossia salp. $y$ che in sloveno dà $i .^{81}$ Tra $i$ prestiti antichi che abbiamo raccolto non c'è nessun esempio di questo genere, si può invece incontrare il sostituto $u$ in múr/val (MŌRUM, REW 5696) e račún (RATIONE, REW 7086). Gli studiosi ritengono che i prestiti con questo sostituto siano meno antichi. ${ }^{82}$ Secondo lo Shevelov, il sostituto slavo per la $o$ del lv. nella posizione non accentata sarebbe $\sigma$, ma il nostro materiale non offre nessun esempio di tale sostituzione..$^{83}$

\section{h) $\breve{u}$ del lc. $>o$ del lv.:}

Nella posizione accentata questa vocale si sostituisce, a quanto sembra, con la 6 dello slavo alpino che in sloveno poi si vocalizza in accordo con l'intonazione e con la

77 Cfr. Bidwell 1961: 113, nota 15. Questo autore menziona soltanto il sostituto 8 , ma fa notare nello stesso tempo la mancanza di esempi per questa vocale (ibid. 115).

78 Cfr. Shevelov 1964: 438. La parola è problematica e presenta parecchie difficoltà d'ordine fonetico (cfr. Šega 1996: 77-79).

79 Cfr. Ramovš 1924: 69; LRA 256-257; Šega 1996: 125-126.

80 Anche per la sillaba non accentata Bidwell parla soltanto del sostituto $\sigma$ con la stessa avvertenza che per la $o$ accentata del latino volgare. V. sopra, nota 77.

81 Si incontrano parecchi esempi di tale sviluppo nei toponimi in -ŌNA (Bidwell, 1961: 116). Cfr. Lamprecht 1987: 45. Doria colloca il passaggio $o>u$ sul territorio dell'Istria già all' inizio del periodo romanzo fondando la sua tesi sulle grafie DUNAT, FURMA ecc. conservate a Parenzo nelle iscrizioni dal quinto o sesto secolo (Doria 1981: 58).

82 Secondo Bidwell, l'esempio POLAE > Pulj non si può paragonare con ROMAE > Rim (come fa più tardi Muljačić, cfr. 1991: 164) perché si tratta di un toponimo "..from the periphery of the area of Slavic settlement, where a non-Slavic population, from whom the name could be borrowed at a later stage, presumably survived longer." (1961: 116).

83 Shevelov si riferisce ai prestiti slavi che risalgono al latino ROMANUS (REW 7371), tra i quali pure sln. rmán (1964: 438). 
sua posizione nella parola: mast/ítil > salp. *msstъ (MŬSTUM, REW 5787), bèč/val (BŬTTIA, REW 1425), vŕč < salp. *vъrčb (ŬRCEUS, REW 9080). Il sostituto y nel caso di kríž < salp. *kryžb (CRŬX CRŪCE, REW 2348) potrebbe rappresentare una prova della pronuncia prolungata della vocale in penultima tonica ${ }^{84} \mathrm{Per}$ la stessa vocale nella posizione non accentata il materiale lessicale studiato presenta sostituti differenti: 3 in skléda, zdéla < salp. *skъdela (SCUTELLA, REW 7756), skódla < salp. ${ }^{*}$ skodъla (SCANDULA, REW 7652), ${ }^{85} o$ in robída (RŬBETUM, REW 7407) e $u$ in lúben (LŬPĨNUS, REW 5170). ${ }^{86}$

\section{i) $\bar{u}$ del lc. $>u$ del lv.:}

Nei prestiti più antichi, la $u$ tonica viene sostituita, nello stesso modo che la $o,{ }^{87}$ con salp. $y>\operatorname{sln}$. $i$ : loč́ka (LACTUCA, REW 4833). ${ }^{88}$ Bezlaj considera antico pure il sostituto $u^{89}$ che non è presente nel nostro materiale. Per lo sloveno ízba si suppone l'assimilazione da una forma romanza proveniente dal lv. ${ }^{*}$ EXTUPA (cfr. EXTUFARE, REW 3108) con la $u$ tonica, ma le forme slave, come avverte Shevelov (1964: 442), fanno pensare piuttosto alla forma di origine $*_{j}$ stb ba. La variante atona di questa vocale non appare nel nostro materiale. ${ }^{90}$

\section{l) dittonghi:}

Per il dittongo lc. $o e$, che in latino volgare passa di solito alla $e$ chiusa o qualche volta alla $e$ aperta, non si trovano esempi nel nostro materiale. ${ }^{91}$ Per il dittongo lc. ae ne abbiamo trovato uno solo, cioè cêsar > salp. *cěsarb (CAESARE, LRA 1472 $\alpha$ ) che dimostrerebbe il passaggio lv. $e>\operatorname{salp} . \check{e}(>\operatorname{sln} . e) .{ }^{92}$ L'esistenza del dittongo latino $a u$,

84 In questo caso la penultima tonica è, per di più, aperta. Di una pronuncia prolungata della vocale si potrebbe parlare eventualmente pure nel caso di jegúlja (ANGUĨLLA, REW 461; all'origine del prestito si trova secondo l'opinione generale la forma ANGULLIA, sorta per metatesi; cfr. Šturm 1927: 76; ESSJ I: 225) che presenta il sostituto $u$. Quest'ultimo è probabilmente alquanto più recente, anche se Bezlaj lo colloca nel periodo dei primi contatti linguistici (1967: 97). Cfr. gúnj (GŬNNA, REW 3919).

85 Cfr. i toponimi Omišalj < AD MUSCULUM e Oprtalj < AD PORTULAM (Skok 1950: I, 24 e 32; Bidwell 1961: 116).

86 In quest'ultimo caso si tratta con molta probabilità di un recente prestito dal croato.

87 Cfr. sopra, g).

88 Succede lo stesso in qualche toponimo, per esempio UTINUM $>$ frl. ant. udino $>$ salp. *v-ydbn $>>$ Viden (Ramovš 1936: 34-35; Bidwell 1961: 116).

89 V. sopra, n. 84.

90 Il Bidwell non fa nessuna distinzione tra gli esiti della $\bar{u}$ lunga e della $\breve{u}$ breve non accentate latine nel latino volgare, ma i suoi esempi illustrano soltanto i sostituti della prima. Cfr. sopra, n. 85 .

91 Possiamo citare soltanto il toponimo POETOVIO $>{ }^{*}$ petouio $>$ salp. ${ }^{*}$ pstujz $>$ sln. Ptuj (cfr. Ramov̌, 1936: 34). Bidwell (1961: 116) si richiama al toponimo bulgaro OESCUS > Iskar che comprova almeno in questo caso lo sviluppo $\mathrm{OE}>e$.

92 Molto probabilmente questo prestito non risale direttamente al latino ma è stato assunto con mediazione germanica (cfr. Gusmani 1986: 63; LRA 1472 $\alpha$ ) 
quello che si è conservato più a lungo di tutti, si manifesta ancora nell'immagine fonica del prestito lôvor (LAURUS, REW 4943). ${ }^{93}$

\section{m) vocale $+r, 1+$ consonante:}

In paleoslavo le vocali davanti alle consonanti $r$ o $l$ in combinazione con un'altra consonante non vengono trattate nello stesso modo che nelle altre posizioni. A parte la metatesi delle consonanti possiamo notare il fatto che le vocali $e$ o $e$ del lv. in questa posizione $(+r$ o $l+$ consonante) si sostituiscono nello salp. con $\check{e}$ che dà in sloveno $e$ : $\check{c}(r) e ́ s ̌ n j a /$ < salp. *črěša (CERĔSEA, REW 1823), brésk/ev/ > salp. *brěsky (PĔRSICA, REW 6427). Nella stessa posizione, la $a$ del lv. si sostituisce con $a$ che dà $a$ in sloveno: ráka (ARCA, REW 611), hláčlel (CALCEA, REW 1495), gradášsal (CARDUUS, REW 168994). Possiamo citare inoltre due esempi di prestiti alquanto più tardi nei quali questo mutamento non si realizza: oltár (ALTARE, REW 381) e járbol (ĀRBŎRE, REW 606).

\section{n) vocale + consonante nasale + consonante:}

La combinazione di una vocale anteriore con $n$ o $m$ seguita da un'altra consonante si sostituisce in paleoslavo con la vocale nasale $e$. L'esito sloveno di questo suono è $e$ : koléda < salp. *kolęda (CALENDAE, REW 1508), méta < salp. *męta (MĔNTA, REW 5504), béka < salp. *bęka (*VĬNCUS, REW 9342). Il prestito brénta (BRENTA, REW 1285) con la consonante nasale conservata appartiene ad una fase più recente ed è stato, come sembra, assimilato soltanto dopo la scomparsa delle vocali nasali nello slavo alpino. Al posto della combinazione di una vocale posteriore con $n$ o $m$ in posizione preconsonantica appare nello slavo alpino la vocale nasale $Q$ che dà in sloveno $o$ : skódla (SCANDULA, REW 7652). Il prestito žúka (JǓNCUS, REW 4619) rappresenta l'unico esempio della sostituzione del gruppo vocale posteriore + consonante nasale + consonante con $u$ : sia che si tratti di una variante dialettale o di un prestito dal croato dove la $o$ paleoslava risulta normalmente in $u .^{95}$ In un solo esempio si può supporre come sostituto per questo gruppo di suoni la $e$ nasale: jegúlja (ANGUIILLA, REW $461^{96}$ ). L'unica spiegazione per questo e per qualche altro degli inaspettati sostituti che abbiamo incontrato è quella proposta dal Bidwell: "Transmission through or contamination by aboriginal (i.e. non-Romanic) Balkan dialects may possibly account for some of the discrepancies noted." (Bidwell 1961: 116).

Secondo il Bidwell (1961: 119-120) nel toponimo Ptuj si manifesterebbe una fase ancora più antica di sostituzione di questa vocale. Si tratterebbe di un toponimo preso in prestito prima della monottongazione slava. Nei prestiti più tardi lo stesso dittongo non è stato monottongato. Cfr. Lamprecht 1987: 45.

Cfr. II.3.

95 Cfr. Šega 1996: 181-182.

96 Cfr. sopra, n. 84. 


\subsection{Consonanti:}

La fricativa sorda $f$ sconosciuta in paleoslavo viene sostituita con $p$. Il nostro materiale offre due esempi di tale sostituzione: pogáča (FǑCACEA, REW 3396) e eventualmente palíska (*FALLIVA, REW 322697). La fricativa sorda $s$ si sostituisce con $\check{s}$ in máša (MÍSSA, REW 5610), gradáš/e/ (CARDUUS, REW 168798) o con $s$ in brósk/va/ (BRASSĬCA, REW 1278).

Alcuni dei prestiti esaminati attestano la sonorizzazione romanza delle consonanti sorde in posizione intervocalica: pogáča (FŎCACEA, REW 3394), robida (RŬBĒTUM, REW 7407), golída (GALLETTA, REW 3656), skléda (SCUTELLA, REW 7756). Sono abbastanza comuni pure le basi con la sorda intervocalica conservata: pôper (PĬPĔRE, REW 6521), rúta (RUTA, REW 7470), konôplja ${ }^{99}$ (*CANNABIA, REW 1598), ločíka (LACTŪCA, REW 4833), prekát (PARRICĀTUS, REW 6524).

Quanto ai cambiamenti articolatori che colpiscono regolarmente non solo le velari davanti alle vocali anteriori ma pure le velari e le dentali davanti a una vocale in iato, dobbiamo rilevare il fatto che fenomeni paralleli agivano tanto in paleoslavo che nel latino volgare. Perciò è difficile stabilire in modo indiscutibile se queste velari si trovavano palatalizzate già nella base romanza al punto che al momento del prestito si sostituirono semplicemente ai suoni paleoslavi apparsi dopo la prima e la seconda patalalizzazione slava, o se sono sorte dai suoni romanzi non palatalizzati come risultato dello sviluppo linguistico slavo. L'esistenza di differenti sostituti anche nei prestiti più antichi si spiega con la palatalizzazione romanza delle costrittive velari davanti alle vocali palatali che può dare risultati differenti secondo l'area di provenienza del prestito: cfr. čéšnja (CERĔSEA, REW 1823) rispetto a cèr (Č̌RRUS, REW 1838). ${ }^{100}$ Ma nella soluzione di questo problema il lessico è di poco aiuto: certi prestiti si sono diffusi molto lontano dall'area dove erano assimilati e, probabilmente, l'areale di molti tra di loro non si potrà purtroppo mai spiegare. L'unica conclusione che possiamo fare è che l'intensità della palatalizzazione delle costrittive velari nelle forme di base romanze doveva differire secondo la parlata di origine e il periodo di

97 Cfr. il frl. falis'cie (NPir. 292). Già nel REW si suppone per questa base l'influsso di *FALLAVISCA, REW 3152.

98 Cfr. II.3.

99 Probabilmente da *CANNAPIA (FEW II: 214), cfr. il friulano cianàipe (NPir. 128).

100 Per questo problema v. sopratutto Skok 1926 e 1928; Ramovš 1926-27; Šturm 1927 o $1932-33$ (in traduzione italiana). Secondo il Ramovš, $c$ e $z$ davanti alle vocali anteriori nei prestiti romanzi antichi rappresenterebbero l'esito della palatalizzazione slava. Questa spiegazione può essere considerata come problematica perché richiederebbe uno sviluppo dei suoni nei prestiti romanzi differente da quello nelle parole di origine slava, come riconosce anche Shevelov: "It (cioè la seconda palatalizzazione slava) is also found in a few loan words with velars followed by front vowels other than $\check{e}$. The assumption is that these words entered Sl/avic/ while the second palatalization of velars was in operation, and became involved in the trend." (Shevelov 1964: 294). Condivide la sua opinione Lamprecht (1987: 46). 
assimilazione e che lo slavo alpino le sostituiva proprio per questa ragione sia con i risultati della prima sia con quelli della seconda palatalizzazione slava.

L'ulteriore sviluppo delle consonanti nei prestiti romanzi antichi non differisce dallo sviluppo normale delle consonanti slave: subiscono tra l'altro la metatesi (cfr. 2.1., paragrafo m), la iodizzazione (konôplja < salp. *konopl'a; cfr. CANNABIA, REW 1598 ${ }^{101}$ ) e varie dissimilazioni (járbol > jámbor; cfr. ARBŎRE, REW 606).

\section{L'adattamento morfologico dei prestiti romanzi antichi in sloveno}

Nel nostro elenco figurano esclusivamente i prestiti sostantivali, eccezion fatta per i casi nei quali si tratta di qualche verbo derivato da un sostantivo. Quest'ultimo rappresenta però già il risultato dell'ulteriore sviluppo del prestito nella lingua ricevente. A nostro avviso, pure il romanismo antico gradáše deve provenire da una forma sostantivale romanza anche se l'unico successore romanzo conosciuto della parola che serviva di base a questo prestito è il verbo italiano antico cardassare. ${ }^{102} \mathrm{I}$ latinismi ed i romanismi antichi nello sloveno conservano di solito il genere della base latina. Dobbiamo però menzionare una caratteristica morfologica comune a un numero considerevole dei prestiti trattati: almeno in una delle sue varianti slave, il prestito appare con la desinenza che lo assegna al gruppo declinazionale in - $u$. L'influsso dei sostantivi con significato simile che appartengono a questo gruppo non deve però essere considerato come la ragione di questo fenomeno ma solo come un fattore promotore stimolato dalla tendenza all'adattamento morfologico dei prestiti ai modelli flessionali della lingua che compie il prestito. Adoperando il termine utilizzato da Gusmani possiamo parlare nel nostro caso del fenomeno di "ipercaratterizzazione suffissale" (Gusmani 1986: 68-69), favorito, come dice l'autore, dall'esistenza di un gruppo di lessemi con significato simile nella lingua ricevente. ${ }^{103}$

\section{Significati dei prestiti antichi nello slavo alpino}

Il processo del prestito consiste nell'imitazione di un segno appartenente al sistema semasiologico della lingua d'origine del prestito. Volendo stabilire le ragioni dell'assimilazione dei latinismi e dei romanismi antichi nello sloveno, dobbiamo prima determinare i campi semantici a cui queste parole appartengono. La classificazione dei campi semantici nei quali si possono incontrare dei residui linguistici appartenenti al sostrato proposta dal Bezlaj non prende in considerazione solo le parole di origine latina o romanza ma abbraccia l'insieme dei termini di origine preslava in sloveno. Risulta da queste indagini che $\mathrm{i}$ residui del sostrato appaiono soprattutto nella

101 Cfr. sopra, n. 99.

102 REW 1687, DELI I: 205. Cfr. Šega 1996: 64-65.

103 Il Bezlaj (1967: 30) osserva che, fin dall'inizio dello sviluppo linguistico indipendente dello sloveno, la derivazione con i mezzi di formazione delle parole esistenti nella lingua si praticava pure nei prestiti. Cfr. per esempio il toponimo Mirje < sln. dialettale mír (MURUS, REW 5764). 
terminologia geografica e pastorizia, nei nomi delle piante industriali e nei termini che servono per indicare oggetti di uso domestico (Bezlaj, 1967: 31).

Un riesame delle ricerche in questo campo dimostra che $\mathrm{i}$ loro risultati non sempre concordano. Evidentemente, le classificazioni non sono state fatte secondo gli stessi criteri ed è per questa ragione che le loro categorie sono difficilmente paragonabili. Facendo una classifica qualsiasi delle parole che stiamo esaminando dobbiamo prendere in considerazione il fatto che il nostro elenco si limita soltanto alle parole incluse nel dizionario dello sloveno letterario (SSKJ). Abbiamo dunque lavorato sulla base di un fondo di parole che non ci permette di fare delle conclusioni definitive sull'intensità dei contatti linguistici in differenti campi semantici. Per questa ragione non ci siamo proposti di fare una propria classificazione: abbiamo invece cercato di stabilire in che misura le parole che stiamo esaminando confermino i risultati delle indagini esistenti. Volendo includerle nei campi semantici menzionati nella classificazione del Bezlaj ci rendiamo conto che per la maggior parte sono nomi di piante industriali e termini per oggetti di uso domestico. Di termini geografici non ne abbiamo trovato neanche uno solo, ma anche i termini della pastorizia sono molto pochi. ${ }^{104}$ La classificazione che meglio corrisponde alla situazione desunta dall'analisi del materiale raccolto, è quella di Mihăescu che si riferisce agli elementi di origine latina non solo in sloveno ma in tutte le lingue slave meridionali. Il Mihăescu (1978: 34-35) ha classificato i latinismi antichi di quest'area in cinque categorie semantiche: 1) flora, 2) fauna, 3) prodotti del lavoro, 4) edilizia e 5) cristianesimo.

In generale si può dire che esistono due tipi di prestiti: quelli che denominano un oggetto o una nozione per la quale non esiste ancora un termine adatto nella lingua ricevente da una parte e dall'altra quelli che sostituiscono termini che esistono o sono esistiti nella lingua ricevente ma hanno smesso di adempiere le loro funzioni. Riesaminando i campi semantici nei quali si possono incontrare i romanismi antichi e le circostanze storiche nel tempo della loro assimilazione, possiamo dire che in quel periodo si trattò di prestiti del primo tipo, di denominazioni per modi sconosciuti di costruzione, tipi sconosciuti di recipienti, nuovi attrezzi, abbigliamenti ed alimenti, tra $i$ quali soprattutto le piante ed $\mathrm{i}$ frutti mediterranei. I prestiti sono legati alla nuova religione, al nuovo sistema sociale e alle attività che gli autoctoni avevano insegnato agli slavi alpini, soprattutto la viticoltura, la pesca e la marineria. Nel nostro elenco figura anche qualche termine mercantile (račún, cfr. RATIŌNE, REW 7086). Secondo la classificazione dei fattori stimolanti dell'assimilazione dei prestiti stabilita da Hope si tratterebbe nello slavo alpino della motivazione estrinseca ("motivation extrinsèque") ossia dei fattori non lessicali dell'assimilazione dei prestiti. ${ }^{105}$ Alla luce di questa

104 La nostra immagine di questo campo semantico è senza dubbio falsata perché abbiamo preso in considerazione solo le parole che si trovano nel dizionario dello sloveno letterario. I termini della pastorizia appartengono per la maggior parte ai dialetti. Cfr. Novak 1975.

105 Si tratta dunque dei prestiti "...dont l'adoption répond au besoin de désigner un objet jusqu'alors inconnu, d'habitude l'adoption simultanée du mot d'emprunt et de l'objet ou le concept dénommé." (Hope 1965: 154). Tra le basi dei nostri prestiti non è trascurabile il numero di quelle che 
constatazione sembra tanto più logico il fatto che dal punto di vista semantico, $i$ latinismi ed i romanismi antichi in sloveno non si allontanano praticamente dalla loro base latina o romanza.

In certi campi semantici la possibilità di assimilazione dei prestiti era più grande che in altri, non solo nel periodo paleoslavo ma anche più tardi. Per indicare lo stesso oggetto o la stessa nozione possiamo trovare perciò in sloveno più di una parola di origine straniera. Spesso tutte provengono dalla stessa base latina sebbene prese in prestito da lingue differenti e in periodi differenti: ${ }^{106}$ prima direttamente nella fase antica, ossia paleoslava, più tardi indirettamente attraverso differenti dialetti tedeschi vicini nei differenti stadi del loro sviluppo. I prestiti più recenti dal tedesco sostituiscono i latinismi antichi, conservati eventualmente in qualche dialetto, allorché il prestito antico dalla stessa base latina sopravvive nelle altre lingue slave che hanno conosciuto una germanizzazione meno intensa. Questo succede per esempio con la base latina MŬSTUM (REW 5783), presa in prestito con la mediazione del medio alto tedesco ( $\sin$. mošt): lo sloveno letterario ha conservato il prestito indiretto dal latino solo nei derivati (mestílec, mastíti, etc.), mentre il sostantivo mest sopravvive a livello dialettale. ${ }^{107}$ Esempi di questo genere confermano l'opinione ripetutamente espressa che lo strato latino dei prestiti in sloveno sia stato ricoperto dai prestiti più recenti appartenenti all'adstrato.

\section{Conclusioni}

Alla fine del nostro contributo vorremmo valutare, quanto ci permette il materiale raccolto, l'intensità dei contatti linguistici slavo-romanzi sul territorio delle Alpi orientali e nell'area nordadriatica nel periodo che ci interessa a paragone con gli altri territori di colonizzazione slava. In base allo studio delle voci appartenenti al nostro fondo di prestiti antichi possiamo parlare di una certa unità sull'intero territorio della colonizzazione slava. La grande maggioranza delle parole latine che servono di base ai prestiti antichi sopravvivono sotto forma di prestiti in più di una lingua slava. Alcune appartengono soltanto allo slavo occidentale o orientale, altre poi si incontrano solo in sloveno e nei dialetti occidentali del croato. Non possiamo dunque nemmeno parlare di basi che sarebbero state assimilate esclusivamente dallo sloveno. Dopo l'esclusione

rappresentano dei prestiti anche dal punto di vista del latino, cioé alcune basi mediterranee (CËRRUS, REW 1838, sln. cèr; LOLIUM, REW 5112, sln. ljúlj/ka/), alcuni grecismi (CANNABIA, REW 9356, sln. konôplja), cfr. FEW ss.vv. Queste parole sono state prese in prestito dalle lingue autoctone, semplicemente perché indicavano dei significati per i quali all'inizio neppure il latino possedeva termini adatti.

106 Questo fatto, notevole in diacronia, è naturalmente di secondaria importanza per lo studio dei singoli prestiti.

107 Plet. I: 574; cfr. anche la forma dialettale mast (Boršt; Cossuta, 1995: 17). 
delle radici che servono di base ai prestiti panslavi, ci restano solo *FAVĪLLA (REW. 3226, sln. palíska) e *PARRICĀTUS (REW 6524, sln. prekát) assunte soltanto dallo sloveno e dai dialetti croati occidentali, SYRICUS (REW 8503, sln. sírek) assimilato in sloveno, croato e serbo e eventualmente *VĬNCUS, REW 9342 ( $\operatorname{sln}$. béka), ${ }^{108}$ un piccolo gruppo di parole dunque, che non ci permette naturalmente di trarre conclusioni sulle eventuali particolarità lessicali nel latino di questo territorio. Tutte le basi latine dei prestiti raccolti hanno però qualcosa in comune: si tratta di parole usate in gran parte dei casi per designare oggetti di uso comune, attrezzi, piante, animali, ecc. ed assimilate nel loro significato latino basico e concreto.

Abbiamo visto che $\mathrm{i}$ latinismi ed i romanismi antichi in sloveno sono molto rari. Possiamo comunque considerare giusta l'opinione che nello slavo alpino i romanismi ed i latinismi fossero molto più numerosi di quello che si può concludere dallo stato attuale della lingua. Il materiale raccolto dimostra che spesso i latinismi e i romanismi assimilati nel periodo paleoslavo venivano sostituiti dai romanismi o - ancora più spesso - dai germanismi più recenti. ${ }^{109}$ Il fatto che i prestiti dalle stesse basi appaiano pure nelle altre lingue slave, rende evidente la complessità della loro storia e dà, in un gran numero di casi, motivo di supporre la mediazione germanica. Quanto al destino del latino sul nostro territorio, dobbiamo comunque convenire con Franco Crevatin quando afferma: "Il quadro complessivo non lascia dubbi: sull' area dell'attuale Slovenia non c'è stato assorbimento di consistenti gruppi latinofoni da parte degli slavi." (Crevatin, 1992: 214). Insieme con i prestiti antichi finora conosciuti e che non compaiono nel Dizionario dello sloveno letterario ma appartengono piuttosto alla sfera dialettale della lingua, gli appellativi assimilati dal latino o da un dialetto romanzo prima dell' inizio del XII secolo non dovrebbero superare il centinaio. Anche con il perfezionamento dell'atlante linguistico sloveno ché renderà il materiale ivi raccolto accessibile ai ricercatori, non possiamo aspettarci un aumento sostanziale di questo numero. Le ricerche in questa direzione possono tuttavia portare alla scoperta di qualche nuovo prestito antico finora sconosciuto che contribuirà alla comprensione di certi fenomeni fonetici e permetterà eventualmente di identificare il centro d'irradiamento per alcune delle basi. Per conseguire una vista d'insieme sui romanismi ed i latinismi antichi in sloveno dovrebbero essere studiati da una parte il lessico sloveno nella totalità, incluso quello dialettale, e anzitutto il vocabolario legato a certe attività artigianali, e dall'altra tutti i termini onomastici di origine latina che rimangono in gran misura non chiariti.

108 Cfr. Šega 1996: 32:34.

109 Il Bezlaj esprime la stessa opinione: "Zdi se, da je v prvih stoletjih po naselitvi igral romanski adstrat bistveno važno vlogo ter ga je šele kasneje začel izpodrivati germanski." (1967: 32). 


\begin{tabular}{ll}
\multicolumn{2}{l}{ ABBREVIAZIONI } \\
aat. & antico alto tedesco \\
cr. & croato \\
frl. & friulano \\
it. & italiano \\
lc. & latino classico \\
Iv. & latino volgare \\
salp. & slavo alpino \\
sin. & sloveno
\end{tabular}

\section{BIBLIOGRAFIA CITATA}

ANS: Arheološka najdišča Slovenije, Ljubljana, 1975.

AO: Alpes orientales $V$. Acta quinti congressus de ethnographia Alpium orientalium tractantis (Graecii Slovenorum, 29.III. 1967-1.IV.1967), Ljubljana, 1969.

Bezlaj 1968-69: France Bezlaj: "Manfred Mende, Romanische Lehnwörter im Slovenischen. Inaugural-Dissertation zur Erlangung des Doktorgrades genehmigt durch die Philosophische Fakultät der freien Universität Berlin.", in: Linguistica, 8/2, 213-218.

Bezlaj 1961: "Die vorslavischen Schichten im slovenischen Namen- und Wortschatz", in: VI. Internationaler Kongress für Namenforschung (München, 24.-28. August 1958) /hgg. von Karl Puchner/, Band II, Verlag der Bayerischen Akademie der Wissenschaften, München, 148-153.

Bezlaj 1967: Eseji o slovenskem jeziku, Ljubljana.

Bezlaj 1969: "Das vorslawische Substrat im Slowenischen", $A O, 19-35$.

Bidwell 1961: Charles E. Bidwell, "The Chronology of Certain Sound Changes in Common Slavic as Evidenced by Loans from Vulgar Latin", in: Word, 17, 105127.

Bratož 1990: Rajko Bratož, Vpliv oglejske cerkve na vzhodnoalpski in predalpski prostor od 4. do 8. stoletja, Ljubljana.

Cossuta 1995: Rada Cossuta, "I romanismi nella terminologia viticola dell'Istria slovena", in: Linguistica, 35/2, 3-36.

Crevatin 1992: Franco Crevatin "Romania disiecta e Romania submersa nell' Adriatico orientale", in: Actes du XVIIIe Congrès International de Linguistique et de Philologie Romanes (Université de Trèves, 1986). I. Romania submersa - Romania nova, Tübingen, 211-226.

Dahmen 1992: Dahmen, "La romanité pannonienne", in: Actes du XVIIIe Congrès International de Linguistique et de Philologie Romanes (Université de Trèves, 1986). I. Romania submersa-Romania nova, Tübingen, 109-114.

DELI: Manlio Cortelazzo, P. Zolli, Dizzionario etimologico della lingua italiana, I-IV, Bologna, 1978-1985.

DLS: Hildegard Striedter-Temps, Deutsche Lehnwörter im Slovenischen, Berlin - Dahlem, 1963. 
Doria 1981: Mario Doria, "Il vocalismo istriano preveneto alla luce della toponomastica medievale e moderna", in: Incontri linguistici, 7, 55-68.

ESSJ: France Bezlaj, Etimološki slovar slovenskega jezika, I-III, Ljubljana, 19761995.

ERHSJ: Petar Skok, Etimologijski rječnik hrvatskoga ili srpskoga jezika, I-IV, Zagreb, 1971-1974.

Ferluga-Petronio 1980: Fedora Ferluga-Petronio, "Il natale nelle lingue slave", in: Linguistica, 20, 131-146.

Ferluga-Petronio 1981: Fedora Ferluga-Petronio, "I nomi delle feste mariane nelle lingue slave", in: Linguistica, 21, 145-163.

FEW: Walther von Wartburg, Französisches Etymologisches Wörterbuch, Bonn, 1922-.

Gaeng 1987: Paul Gaeng, "Variétés régionales du latin parlé: le témoignage des inscriptions", in: Latin vulgaire - latin tardif. Actes du Ier Colloque international sur le latin vulgaire et tardif (Pécs, 2-5 septembre 1985) (ed. Jozsef Herman), Tübingen, 77-86.

Grad 1965: Anton Grad, "Importance de quelques toponymes slovènes pour la géographie linguistique romane", Actes du Xe Congrès de Linguistique et Philologie Romanes (Strasbourg, 1962), III, Paris, 1176-1184.

Grad 1969: Anton Grad, "Contributo al problema della palatalizzazione delle gutturali $c, g$ davanti ad $a$ in friulano", in: Atti del congresso internazionale di linguistica $e$ tradizioni popolari, Gorizia - Udine - Tolmezzo, 101-106.

Grafenauer, 1954: Zgodovina slovenskega naroda, I, Ljubljana.

Grafenauer 1969: Bogo Grafenauer, "Die Kontinuitätsfragen in der Geschichte des altkanatanischen Raumes", in: $A O$, 55-85.

Grafenauer 1988: "O Pavlu Diakonu in začetkih Slovencev v novi domovini", in: Pavel Diakon: Zgodovina Langobardov, Maribor, 295-422.

Gusmani 1986: Saggi sul interferenza linguistica, Firenze.

Herman 1976: Joseph Herman, "La latinité des provinces de l'empire romain. Problèmes de sociolinguistique", in: XIV Congresso internazionale di linguistica e filologia romanza (Napoli, 15-20 aprile 1974), II, Napoli - Amsterdam, 7-15.

Hope 1965: Thomas E. Hope, "L'interprétation des mots d'emprunt et la structure lexicale", in: Actes du Xe Congrès international de Linguistique et Philologie Romanes (Strasbourg, 1962), I, Paris, 149-155.

Iliescu 1987: Maria Iliescu, "Ce que nous apprend le vocabulaire de base du latin sur la diversification lexicale des langues romanes", in: Latin vulgaire - latin tardif. Actes du Ier Colloque international sur le latin vulgaire et tardif (Pécs, 2-5 septembre 1985), Tübingen, 109-119.

Korošec 1979: Paola Korošec, Zgodnjesrednjeveška arheološka slika karantanskih Slovanov, I-II, Ljubljana.

Kos 1956: "O imenih nekaterih krajev v Slovenskem primorju", in: Zbornik Primorske založbe Lipa, Koper, 7-26.

Kos 1985: Srednjeveška kulturna, družbena in politična zgodovina Slovencev. Izbrane razprave, Ljubljana.

Lamprecht 1987: Arnošt Lamprecht, Praslovanština, v Brně.

Logar 1965: Tine Logar, "O nastanku slovenskih narečij", in: Jezik in slovstvo, 10/1, 26. 
LLMAJ: Lexicon latinitatis medii aevi Iugoslaviae, I-VII, Zagrabiae MCMLXIX MCMLXXVIII.

LRA: Luciano Rocchi, Latinismi e romanismi antichi nelle lingue slave meridionali, Udine, 1990.

Mihăescu 1978: Haralambie Michaescu, La langue latine dans le sud-est de l'Europe, Bucuresti - Paris.

Muljačić 1978: Žarko Muljačić, "Lingue romanze e lingue slave", in: XIV Congresso internazionale di linguistica e filologia romanza (Napoli, 15-20 aprile 1974), I, Napoli - Amsterdam, 407-421.

Muljačić 1991: Žarko Muljačić, "Sullo status linguistico dell'istrioto medievale", in: Linguistica, 31, 155-170.

Nahtigal 1952: Rajko Nahtigal, Slovanski jeziki, Ljubljana.

Novak 1975: Vilko Novak, "Predromanske, romanske in germanske besede $v$ slovenskem gorskem pastirstvu", in: Linguistica, 15, 109-114.

NPir: Giulio Andrea Pirona - Ercole Carletti - Giov. Batt. Corgnali, Il Nuovo Pirona. Vocabolario friulano, Udine, 1935.

Plet. M. Pleteršnik, Slovensko-nemški slovar, I-II, Ljubljana, 1894-95.

Popović 1960: Ivan Popović, Geschichte der serbokroatischen Sprache, Wiesbaden.

Ramovš 1924: Historična gramatika slovenskega jezika. II. Konzonantizem, Ljubljana.

Ramovš 1926-27: "O prvotnih južsl. substitucijah za balk.-lat. $k, g$ pred $e, i "$, in: Južnoslovenski filolog, 6, 153-165.

Ramovš 1936: Fran Ramovš, Kratka zgodovina slovenskega jezika, Ljubljana.

REW: Wilhelm Meyer-Lübke, Romanisches etymologisches Wörterbuch, Heidelberg 1935.

RLS: Manfred Mende, Romanische Lehnwörter im Slovenischen. Inaugural-Dissertation zur Erlangung des Doktorgrades genehmigt durch die Philosophische Fakultät der Freien Universität Berlin (Fak. No 263), Berlin (s.a.).

Shevelov 1964: George Y. Shevelov, A prehistory of Slavic. The historical phonology of common Slavic, Heidelberg.

Skok 1921: Petar Skok, "Oglej i Celje", in: $\check{C} J K Z, 3 / 1-2,24-32$.

Skok 1926: Petar Skok, "Zur Chronologie der Palatalisierung von $c, g, q u, g u$ vor $e, i$, $y, i$ im Balkanlatein", in: $Z R P h, 46,385-410$.

Skok 1928: Petar Skok, "O slovenskoj palatalizaciji sa romanističkog gledišta", in: Južnoslovenski filolog, 8, 38-64.

Skok 1931: Petar Skok, "Iz slovenačke toponomastike", in: $\breve{C} J K Z, 8,118-125$.

Skok 1940: Petar Skok, Osnovi romanske lingvistike, Zagreb

Skok 1950: Slavenstvo i romanstvo na jadranskim otocima, Zagreb, 1950

Skubic 1981: Mitja Skubic, "La langue des inscriptions latines en Slovénie", in: Linguistica, 21, 277-298.

SSKJ: Slovar slovenskega knjižnega jezika, Ljubljana, 1994.

Stefenelli 1992: Arnulf Stefenelli, Das Schicksal des lateinischen Wortschatzes in den romanischen Sprachen, Passau.

SVI: France Bezlaj, Slovenska vodna imena, I-II, 1956-1961.

Šega 1996: Agata Šega, Starejši latinski jezikovni fond v slovenščini, tesi di master, Ljubljana. 
Štrekelj 1890: Karel Štrekelj, "Beiträge zur slavischen Lehnwörterkunde I", in: ASPh, $12,451-74$.

Štrekelj 1892: Karel Štrekelj, "Beiträge zur slavischen Lehnwörterkunde II", in: ASPh, $14,512-55$.

Šturm 1927: Fran Šturm, "Refleksi romanskih palataliziranih konzonantov v slovenskih izposojenkah", in: $\breve{C} J K Z, 6,45-85$.

Šturm 1928: Fran Šturm, "Romanska lenizacija medvokaličnih konzonantov in njen pomen za presojo romanskega elementa v slovenščini", in: $\breve{C} J K Z, 7,21-46$.

Šturm 1932-33: "Riflessi di consonanti palatali neolatine", in: Ce fastu, 8, 217-21, 265-270; Ce fastu, 9, 1-9, 57-62, 119-25.

Tekavčić 1987: Pavao Tekavčić, "Vojmir Vinja, Jadranska fauna. Etimologija i struktura naziva, I-II, Split, Logos, 1986: I. vol. pp. 5-504, II. vol. pp. 1-508.", Linguistica, 27, 149-158.

Zgodovina: Zgodovina Slovencev (ed. Bogo Grafenauer), Ljubljana, 1979.

Povzetek

PRISPEVEK K POZNAVANJU STAREJŠIH LATINIZMOV IN ROMANIZMOV V SLOVENŠČINI

V pričujočem prispevku avtorica povzema ugotovitve magistrske naloge Starejši latinski jezikovni fond v slovenščini (mentor prof. Mitja Skubic, somentorica prof. Alenka Šivic-Dular), ki jo je v januarju 1997 zagovarjala na Filozofski fakulteti v Ljubljani. Na začetku na kratko prikaže dosedanje ugotovitve zgodovinskih ved o romanizaciji, kontinuiteti in jezikovnih stikih na območju vzhodnih Alp in severnega Jadrana v praslovanskem obdobju, nato pa se posveti analizi zbranega jezikovnega materiala, to je približno 70 starejših latinizmov in romanizmov, vključenih v Slovar slovenskega knjižnega jezika (SSKJ), ki izvirajo iz latinskih apelativov in so bili zelo verjetno prevzeti nekako do konca 11. stoletja.

Prvi del analize vsebuje pregled alpskoslovanskih substitutov za različne latinske glasove, v katerem avtorica ugotovitve dosedanjih raziskav, ki so nastale zlasti na podlagi toponomastičnega gradiva, dopolnjuje $z$ rezultati preučevanja zbranih izposojenk. Za vokalizem starih izposojenk so značilne zlasti substitucije klas. lat. nagl. $\bar{e}$, vulg. lat. $e>$ alp. sla. in sln. $i$; klas. lat. $\bar{u}$, vulg. lat. $u>$ alp. sla. $y>\sin . i$, klat. a, vulg. lat. $a \vee$ predzadnjem zlogu $>$ alp. sla. $\breve{a}>\operatorname{sln} . o$, pomembne pa so tudi tiste, ki pričajo o obstoju tako imenovanih ultrakratkih vokalov, na primer máša (MĬSSA, REW 5610), vŕč (ŬRCEUS, REW 9080), in nosnih samoglasnikov, na primer méta (MĚNTA, REW 5504) proti míza (MENSA, REW 5610). Pri konzonantih opozarja na dvojnost substitutov za latinske oziroma romanske palatalizirane glasove kakor v čéšnja (CERASEA, REW 1823) za razliko od cèr (CĚRRUS, REW 1838), kar po njenem mnenju kaže na različne stopnje palataliziranosti romanskih glasov v izhodiščnih oblikah. Slednje pričajo tudi o tem, da so bile nekatere izposojenke prevzete iz osnov s sonoriziranimi medvokalnimi nezvenečimi zaporniki, druge pa ne, kakor se kaže v opoziciji rúta (RUTA, REW 7470) proti golida (GALLETA, REW 3656).

V drugem delu analize avtorica ugotavlja, da so stari latinizmi in romanizmi v slovenščini (gre izključno za samostalniške izposojenke) docela prilagojeni morfološkemu sistemu ciljnega jezika, njihova pomenska analiza pa pripelje do spoznanja, da gre $v$ tem zgodnjem obdobju za zunanjo motivacijo izposoje: opraviti imamo predvsem $z$ rastlinskimi in živalskimi imeni, izrazi s področja krščanstva in stavbarstva in $z$ besedami, ki poimenujejo izdelke in predmete za vsakdanjo rabo, kar pomeni, da gre za pojme, ki so jih alpski Slovani spoznali po prihodu v novo domovino in zanje $v$ jeziku sprva niso imeli ustreznih poimenovanj. 